\title{
Synthesis of diacyl amino acid surfactant and evaluation of its potential for surfactant-polymer flooding
}

\author{
Hongmei Ren - Changxin Shi $\cdot$ Shemin Song • \\ Qingqiao Zeng $\cdot$ Yuzhen Zhang
}

Received: 4 July 2014/Accepted: 18 November 2014/Published online: 4 June 2015

(c) The Author(s) 2015. This article is published with open access at Springerlink.com

\begin{abstract}
Diacyl amino acid surfactant was prepared using lauroyl chloride and L-lysine. The structure of the synthesized surfactant was confirmed by infrared spectroscopy and mass spectroscopy. The ultralow interfacial tension could be achieved when the diacyl amino acid surfactant is mixed with sulfonate in the presence of polymer as well as absence of polymer. Core flood experiments showed that $17.8 \%$ additional oil could be recovered by injection of 0.3 pore volume of surfactantpolymer solution, making the total oil recovery reach $66.1 \%$.
\end{abstract}

Keywords Synthesis - Diacyl amino acid surfactant . Ultralow interfacial tension · Flooding

\section{Introduction}

Surfactant is an important type of fine chemical product, which is often dubbed as "the industrial monosodium glutamate" and is consumed in large quantities [1]. However, the water and soils will be contaminated when a large amount of surfactants are discharged into them. In recent years, to meet requirement of the environmental protection

\section{H. Ren}

East China University of Science and Technology,

200237 Shanghai, China

H. Ren · C. Shi $(\bowtie) \cdot$ Q. Zeng · Y. Zhang

Exploration and Development Research Institute of Huabei

Oilfield Company, 062552 Renqiu, Hebei, China

e-mail: cxshi@qq.com

S. Song

Development Department of Huabei Oilfield Company,

062552 Renqiu, Hebei, China and biological safety, more attention has been paid to develop and use the environment-friendly surfactants.

Amino acid-based surfactants are a class of surfactants with excellent biocompatibility, emulsifying ability, low toxicity, low irritation, as well as antimicrobial properties $[2,3]$. They are now very commonly used in house cleaning products $[4,5]$, food industry, pharmaceutical applications, and foam flotation, textile and leather industry, but seldom in oil recovery.

It is well known that, the oil recovery can be improved by injecting surfactants, alkalis or polymers to displace the residual oil that trapped in the reservoir. Nevertheless, the alkali containing flooding can cause many problems, such as erosion, reduction of productivity and the shortening of pump checking $[6,7]$. To avoid using alkali, we tend to develop alkali-free surfactant-polymer flooding system. One of the structural characteristics of the flooding using surfactants is branched. This is because branched surfactants often show a better interfacial tension lowering ability and effectiveness than those with straight-chain alkyl groups $[8,9]$. Low interfacial tension is necessary to mobilize the trapped oil [10]. The conventional surfactants are often single tailed. Here we designed and prepared a double-chain amino acid surfactant diacyl amino acid sodium (DAAS), and evaluated its potential in surfactantpolymer flooding. It should be mentioned that, one of its chains could be seen as a side chain.

\section{Experimental}

Chemicals

Lauroyl chloride, sodium hydroxide, and acetone (AR grade) were purchased from West Asia chemical reagent 
Company. L-Lysine was purchased from Guangfu Fine Chemicals Research Institute. Hydrolyzed polyacrylamide (HPAM), degree of hydrolysis is $22.8 \%$ with a molecular weight of $2.4 \times 10^{7}$, was obtained from Hengju company. Daqing petroleum sulfonate (DPS) was offered by Daqing refinery factory. It was washed and purified by washing with petroleum ether and ethanol to remove the unreacted oil and salts. The crude oil used in this experiment was extracted from Jing11 oil well. The density of the crude oil is $0.81 \mathrm{~kg} / \mathrm{m}^{3}$, the water used in the experiment is the injection water, its salinity is $10,954 \mathrm{mg} / \mathrm{L}$.

\section{Synthesis}

The amino acid surfactant was synthesized according to the [1].The synthetic route was presented in Scheme 1. Acetone $(50 \mathrm{ml})$, water $(25 \mathrm{ml})$ and L-lysine $(7.3 \mathrm{~g})$ were added to a $500 \mathrm{ml}$ three-necked round-bottomed flask equipped with a mechanical stirrer. The mixture was stirred while $32.76 \mathrm{~g}$ lauroyl chloride and $10 \%$ (wt) sodium hydroxide solution were added drop wise. Then, the mixture was vigorously stirred for $4 \mathrm{~h}$ in an ice bath while the $\mathrm{pH}$ was kept near 9 , followed by overnight standing at room temperature. The mixture was placed under vacuum to remove the solvent, then the hydrochloric acid was added to adjust the $\mathrm{pH}$ value to 2 . The obtained product was separated by sucking filtration and washing with deionized water until neutral. The product was then washed three times with petroleum ether and dried under vacuum. The obtained amino acid was dispersed in deionized water at $80{ }^{\circ} \mathrm{C}$ and neutralized with an equal molar quantity of sodium hydroxide (mass concentration was $10 \%$ ). The final product was purified by sucking filtration, drying and recrystallization with ethanol.

\section{Characterization}

The authors characterized the surfactant that had been synthesized in-house using IR and MS.

Infrared spectra were recorded as $\mathrm{KBr}$ pellets on a $\mathrm{Ni}$ colet 8700 FT-IR spectrometer in the scanning range of $4,000-500 \mathrm{~cm}^{-1}$. The $\mathrm{KBr}$ pellets were prepared by gently mixing $1 \mathrm{mg}$ of the dried sample with $100 \mathrm{mg}$ of the dried KBr. MS analysis was carried out on a Waters Quattro Premier XE in a positive mode with an $\mathrm{m} / \mathrm{z}, 50-1,000$ scanning range using methanol as a mobile phase. The operating parameters were set as follows: capillary voltage,
$3.5 \mathrm{kV}$; cone voltage, $30 \mathrm{~V}$; source temperature, $120{ }^{\circ} \mathrm{C}$; desolvation temperature, $150{ }^{\circ} \mathrm{C}$; desolvation gas flow rate, $600 \mathrm{~L} / \mathrm{h}$. The sample solution was prepared by dissolving $10 \mathrm{mg}$ of the sample in $10 \mathrm{~mL}$ of the mobile phase, then injected into the mass detector. Interfacial tensions were measured at $54{ }^{\circ} \mathrm{C}$ (reservoir temperature) using a TX550A spinning-drop tensiometer at a rotation speed of 5,000 rpm. The measurement time was $120 \mathrm{~min}$. The sample was dissolved in the injection water, then injected into a sample tube using a syringe. Core flood test was performed on a sand-packed core of diameter $2.5 \mathrm{~cm}$ and length $50 \mathrm{~cm}$. The permeability of the core was $248.6 \mathrm{mD}$ with the pore volume of $74.6 \mathrm{ml}$. The porosity of the core was obtained by dividing the pore volume by the volume of the packed core. Its value was $30.41 \%$. The oil saturation was calculated by dividing the volume of the oil that was saturated into the core by the pore volume. Its value was $81.1 \%$.

\section{Results and discussion}

\section{Infrared spectroscopy of DAAS}

The infrared spectrum of DAAS is shown in Fig. 1. The bands at 3,324 and $1,421 \mathrm{~cm}^{-1}$ are due to $\mathrm{N}-\mathrm{H}$ stretching vibrations. The band at $2,955 \mathrm{~cm}^{-1}$ is attributed to antisymmetric $\mathrm{CH}_{3}$ stretching vibrations while the band at $2,920 \mathrm{~cm}^{-1}$ can be assigned to antisymmetric $\mathrm{CH}_{2}$ stretching vibrations. The band observed at $2,850 \mathrm{~cm}^{-1}$ can be attributed to the symmetric $\mathrm{CH}_{2}$ stretching vibrations. The absorption at $1,641 \mathrm{~cm}^{-1}$ is due to the amide band whereas the band at $1,557 \mathrm{~cm}^{-1}$ can be assigned to $\mathrm{C}-\mathrm{N}$ stretching and $\mathrm{N}-\mathrm{H}$ bending vibrations.

\section{ESI-MS spectra of DAAS}

The positive ion ESI-MS spectra of DAAS are displayed in Fig. 2. The peak at $m / z=511.4$ corresponds to $[\mathrm{M}-\mathrm{Na}+\mathrm{H}]^{+}$where $\mathrm{m} / \mathrm{z}=512.4$ corresponds to $[\mathrm{M}-\mathrm{Na}+2 \mathrm{H}]^{+}$. The minor peak at $\mathrm{m} / z=329.2$ signifies L-lysine is mono-substituted.

\section{Interfacial tension behavior}

Figure 3 shows the interfacial tension between Jing11 crude oil and injection water. It is observed that neither the DAAS nor the DPS could reduce the interfacial tension to

Scheme 1 Synthetic route of the DAAS

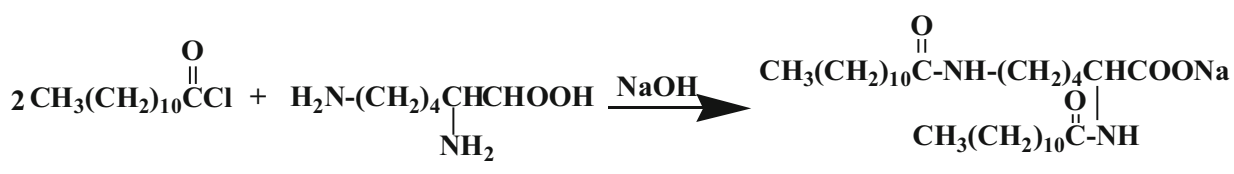


Fig. 1 IR spectra of DAAS

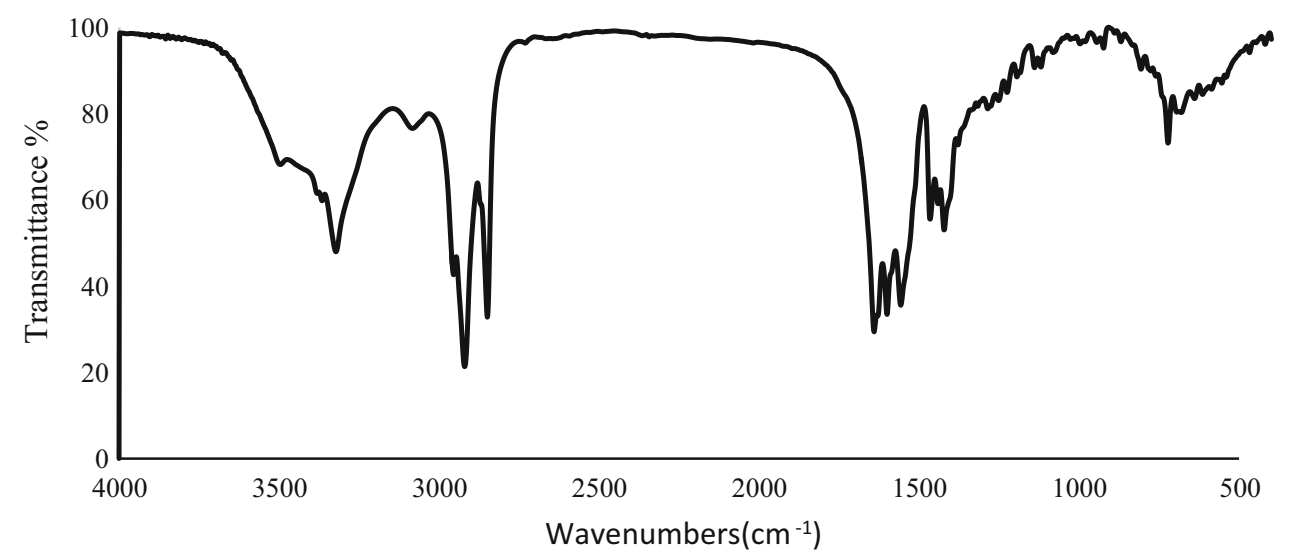

Fig. 2 ESI-MS spectra of DAAS

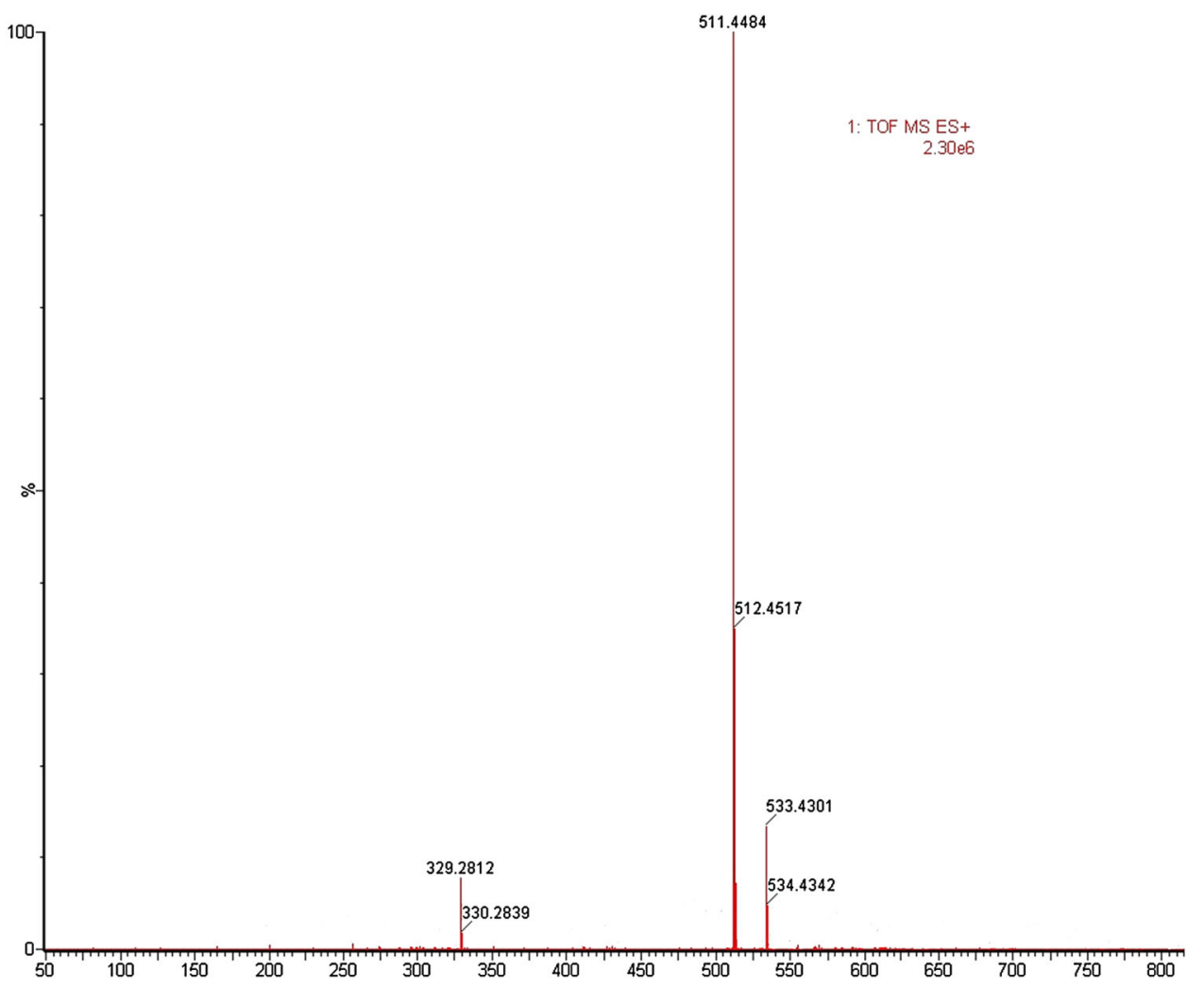

Fig. 3 The lowest interfacial tension between crude oil and injection water

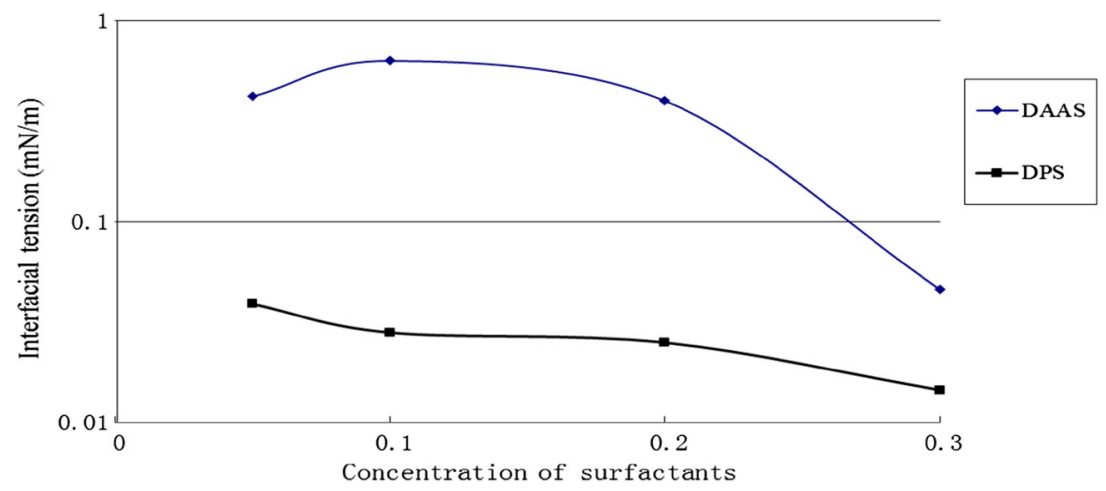

$\checkmark$

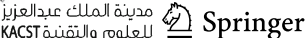


Table 1 The lowest interfacial value of the mixed surfactants

\begin{tabular}{lll}
\hline $\begin{array}{l}\text { DAAS concentration } \\
(\%)\end{array}$ & $\begin{array}{l}\text { DPS concentration } \\
(\%)\end{array}$ & $\begin{array}{l}\text { Interfacial tension } \\
(\mathrm{mN} / \mathrm{m})\end{array}$ \\
\hline 0.05 & 0.025 & $2.81 \times 10^{-3}$ \\
0.05 & 0.05 & $1.07 \times 10^{-3}$ \\
0.05 & 0.1 & $1.1 \times 10^{-4}$ \\
0.1 & 0.05 & $1.28 \times 10^{-2}$ \\
0.1 & 0.1 & $3.18 \times 10^{-3}$ \\
0.1 & 0.2 & $1.07 \times 10^{-3}$ \\
0.15 & 0.15 & $1.08 \times 10^{-3}$ \\
0.2 & 0.1 & $6.63 \times 10^{-4}$ \\
\hline
\end{tabular}

ultralow values. It should be noted that, the DAAS can lower the interfacial tension to a magnitude of $10^{-2} \mathrm{mN} / \mathrm{m}$ at the concentration of $0.3 \%$ (mass ratio). However, when DAAS was mixed with DPS, the ultralow interfacial tension could be reached in the absence of alkaline agents at a wide range of concentrations (see Table 1).

It is evident from Table 1 that the two surfactants showed the synergism in interfacial tension reduction. A possible reason may be that the interfacial activity of binary surfactant mixtures depends on their adsorption at the oil-water interface. And the adsorption is correlated with the tightness of mixed micellar structure [11]. For the DAAS and DPS molecules, their hydrophilic-lipophilic balance (HLB value, the DAAS has a value of 15.56, while for the conventional surfactants, the value is generally around 10) differences cause the different insertion depth at the oil/water interface, which has benefit of reducing repulsion between the polar groups [12] and then influences the tightness of the mixed micellar structure. The compact mixed micellar structures are easy to adsorb at the oil/water interface and have a relatively higher adsorption amount, so the interfacial activity is improved.

In the surfactant polymer flood process, the addition of water-soluble polymer can expand swept volume by increasing the water viscosity and decreasing water-oil mobility ratios [13]. However, it often has a negative effect on the interfacial tension. In view of the interfacial tension factors, the formulation $(0.05 \%$ DAAS $+0.1 \%$ DPS $)$ was chosen to investigate the effect of the polymer (HPAM) on the interfacial tension. The concentration of the polymer was $1,500 \mathrm{mg} / \mathrm{L}$. The results are presented in Fig. 4.

Figure 4 reveals that the interfacial tension increased in the presence of polymer, but still lower than $10^{-2} \mathrm{mN} / \mathrm{m}$. A possible reason for the interfacial increase is that there were hydrophobic domains existed in the polymer solution. The surfactant would adsorb in the hydrophobic domains when added into the solution and formed mixed micelles with hydrophobic chains [14]. Thus, the surfactant adsorption amount at the oil/water interface was cut, which resulted in the interfacial tension decrease. Besides, the
Fig. 4 Dynamic interfacial tension between oil and water

Fig. 5 PV injected
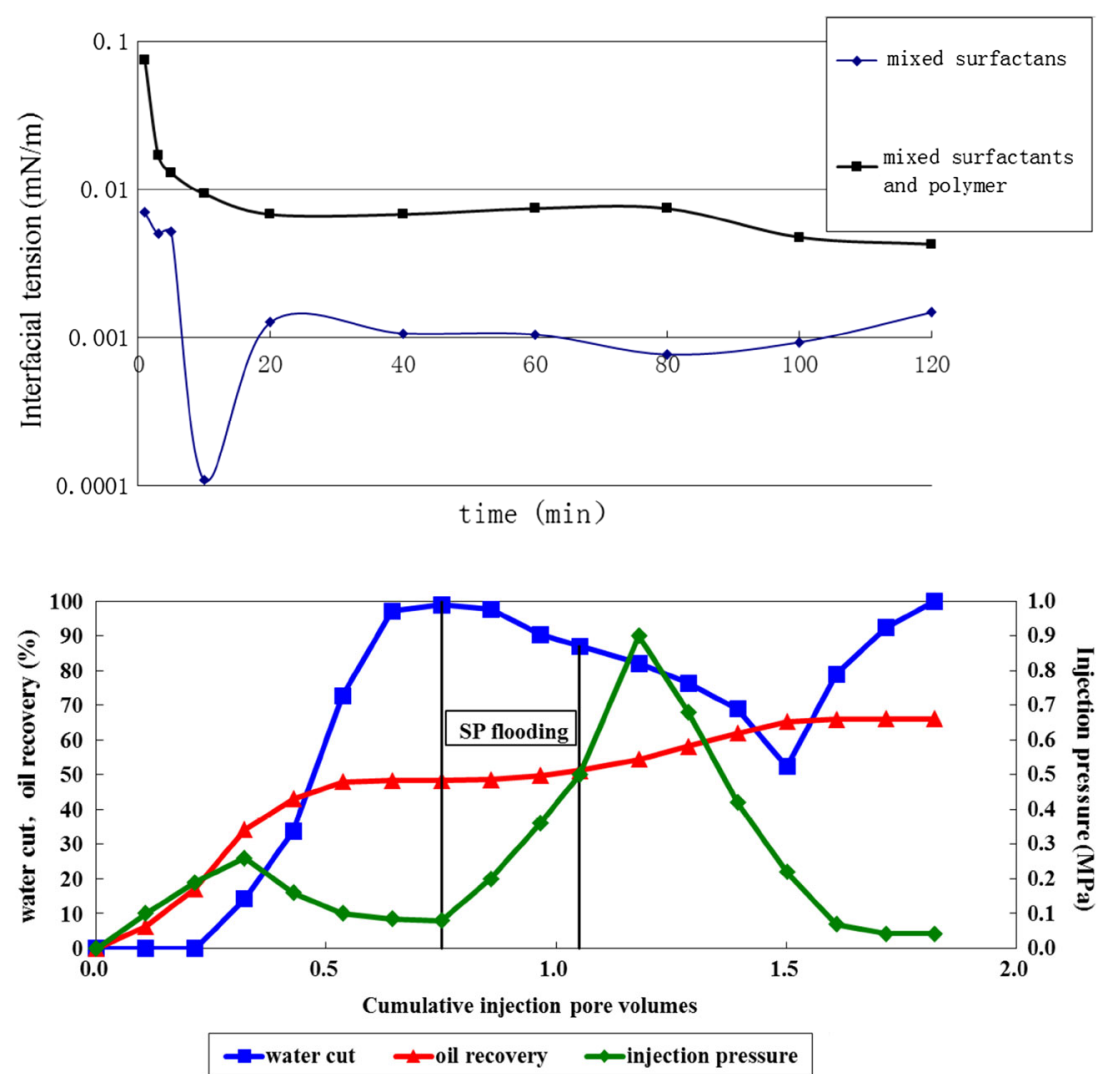
reason for the sharp deviation of the mixed surfactant system at early times is: the oil droplet was first elongated, then broken up into several smaller droplets. The retraction of the droplet caused the interfacial tension increase.

\section{Flooding experiments}

The permeability of the major reservoirs is around $250 \mathrm{mD}$, so a sand-packed core with a permeability of $248.6 \mathrm{mD}$ was prepared. The core was first evacuated with a vacuum pump and saturated with injected water. The permeability of the core to water was calculated by measuring the pressure and the flow rate in accordance with Darcy's law. After that, the core was placed in an oven at $54{ }^{\circ} \mathrm{C}$ and flooded with oil until no extra water was produced. After the oil flooding, the core was aged for $24 \mathrm{~h}$ in the oven at $54{ }^{\circ} \mathrm{C}$ and then was flooded with injected water until the water cut reached $98 \%$. Finally, 0.3 pore volume (PV) of surfactant-polymer solution $(0.15 \%$ of surfactant and $1500 \mathrm{mg} / \mathrm{L}$ of polymer) was injected followed by water injection to reach a water cut of about $98 \%$. The injection rate was kept at $0.4 \mathrm{ml} / \mathrm{min}$ during the experiment. The results are shown in Fig. 5.

As shown in Fig. 5, with the increase of injected pore volumes, the oil recovery increased gradually. Meanwhile, the water ratio rose constantly until it reached $98 \%$ before the surfactant-polymer slug injection. The oil recovery by water flooding was $48.3 \%$. After the injection of surfactant-polymer slug of $0.3 \mathrm{PV}$, the pressure increased rapidly, this is because the slug entered the higher permeability zone, which caused the increase of water flow resistance. The pressure subsequently decreased with the increased oil recovery. The incremental oil recovery was $17.8 \%$ above the water flood, making the total oil recovery reach $66.1 \%$.

\section{Industrial significance}

It is already showed that amino surfactants could be used as oil displacement agents, but different crude oil needed different surfactant formations. The researchers could use other type of surfactants to mix with the synthesized amino surfactant to meet the demand. In the same time, the researchers could try using other amino acids to prepare some novel surfactants.

\section{Conclusions}

Diacyl amino acid sodium was prepared and its structure was confirmed. The single use of the surfactant cannot reduce the interfacial tension to ultralow. When combined with sulfonate, the mixed binary surfactant can lower the interfacial tension to ultralow in the presence of polymer. Core flood test showed that the surfactant-polymer can enhance the oil recovery by $17.8 \%$ over the water flood.

Acknowledgment This study was funded by key technical research and application program of increasing and stable 8 million tons of oil production in Huabei oil field (No. 2014E-35-7).

Open Access This article is distributed under the terms of the Creative Commons Attribution 4.0 International License (http:// creativecommons.org/licenses/by/4.0/), which permits unrestricted use, distribution, and reproduction in any medium, provided you give appropriate credit to the original author(s) and the source, provide a link to the Creative Commons license, and indicate if changes were made.

\section{References}

1. Qiao WH, Zheng ZB, Peng H, Lu S (2012) Synthesis of and properties of three series amino acid surfactants. Tenside Surf Deterg 49:161-166

2. Soo EL, Salleh AB, Basri M, Rahman RNEA, Kamaruddin K (2004) Response surface methodological study on lipase-catalyzed synthesis of amino acid surfactants. Process Biochem 39:1511-1518

3. XU BC, Li JJ (2006) N-Acyl amino acid surfactants. In: Proceedings of the ninth international conference on surfactant and detergent, Shanghai

4. Infante MR, Perez L, Pinazo A, Clapes P et al (2004) Amino acid-based surfactants. C R Chimi 7:583-592

5. Xia JD, Xia YM, Nnanna IA (1995) Structure-function relationship of acyl amino acid surfactants: surface activity and antimicrobial properties. J Agric Food Chem 43:867-871

6. Hou JR, Lui ZC, Zhang SF, Yue XA, Yang JZ (2005) The role of viscoelasticity of alkali/surfactant/polymer solutions in enhanced oil recovery. J Pet Sci Eng 47:219-235

7. Jing JL, Tang S, Yu T, Gai YJ (2014) Effect of HPAM in ASP flooding wastewater on scaling. Desalin Water Treat 52:6930-6935

8. Guo WK, Yang ZY, Wu XL, Zhang GY, Wang HF (2006) New type weak alkali surfactant applied to tertiary oil recovery. ACTA Pet Sin 5:75-78

9. Rosen MJ, Wang HZ, Shen PP, Zhu YY (2005) Ultralow interfacial tension for enhanced oil recovery at very low surfactant concentrations. Langmuir 21:3749-3756

10. Chan KS, Shan DO (1980) The molecular mechanism for achieving ultra-low interfacial tension minimum in a petroleum sulfonate/oil brine/system. J Disper Sci Technol 1:55-95

11. Liu DX, Huang HD, Wu YH, Wang YF (1997) Effects of surface activity of nonionic surfactants on POS and its combined system. J Jianghan Pet Inst 14:51-54

12. Huang HD, Chen YM, Chen Y, He J, Zhang Q (2009) Interfacial activities of petroleum carboxylate and alkylbenzene sulfonate combined system with and without weak alkaline. Oilfield Chem 26:187-190

13. Wang DM, Cheng JC, Qing YY, Gong WC, Li Q, Chen FM (2000) Viscous-elastic polymer can increase microscale displacement efficiency in cores. SPE Annual Technical Conference and Exhibition, Dallas

14. Biggs S, Selb J, Candau F (1992) Effect of surfactant on the solution properties of hydrophobically modified polyacrylamide. Langmuir 8:838-847

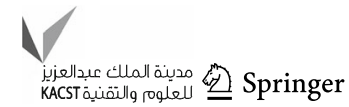

